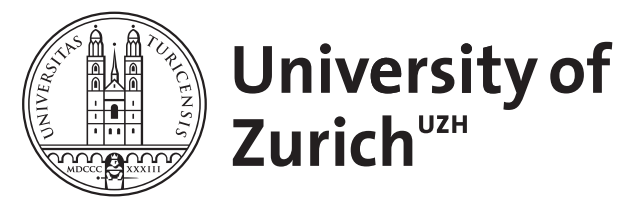

\title{
Hot spots of mutualistic networks
}

Gilarranz, Luis J ; Sabatino, Malena ; Aizen, Marcelo A ; Bascompte, Jordi

\begin{abstract}
Incorporating interactions into a biogeographical framework may serve to understand how interactions and the services they provide are distributed in space. We begin by simulating the spatiotemporal dynamics of realistic mutualistic networks inhabiting spatial networks of habitat patches. We proceed by comparing the predicted patterns with the empirical results of a set of pollination networks in isolated hills of the Argentinian Pampas. We first find that one needs to sample up to five times as much area to record interactions as would be needed to sample the same proportion of species. Secondly, we find that peripheral patches have fewer interactions and harbour less nested networks - therefore potentially less resilient communities - compared to central patches. Our results highlight the important role played by the structure of dispersal routes on the spatial distribution of community patterns. This may help to understand the formation of biodiversity hot spots.
\end{abstract}

DOI: https://doi.org/10.1111/1365-2656.12304

Posted at the Zurich Open Repository and Archive, University of Zurich

ZORA URL: https://doi.org/10.5167/uzh-120825

Journal Article

Accepted Version

Originally published at:

Gilarranz, Luis J; Sabatino, Malena; Aizen, Marcelo A; Bascompte, Jordi (2015). Hot spots of mutualistic networks. Journal of Animal Ecology, 84(2):407-413.

DOI: https://doi.org/10.1111/1365-2656.12304 


\title{
Hotspots of Mutualistic Networks
}

\author{
Luis J. Gilarranz ${ }^{1 *}$, Malena Sabatino ${ }^{2,3}$ \\ Marcelo A. Aizen ${ }^{2}$, and Jordi Bascompte ${ }^{1}$
}

RunNing HeADLine: Hotspots of Mutualistic Networks.

${ }^{1}$ Integrative Ecology Group, Estación Biológica de Doñana, CSIC

Calle Américo Vespucio s/n, 41092 Sevilla, Spain

${ }^{2}$ Laboratorio Ecotono-CRUB

Universidad Nacional del Comahue and INIBIOMA

Pasaje Gutierrez 1125, 8400 San Carlos de Bariloche, Río Negro, Argentina

${ }^{3}$ EEA [Estación Experimental Agropecuaria] Balcarce

INTA [Instituto Nacional de Tecnología Agropecuaria]

CC 276, 7620 Balcarce, Buenos Aires, Argentina

*To whom correspondence should be addressed. E-mail: lj.gilarranz@ebd.csic.es, Phone: $\quad+34$ 954466700, Fax: +34954621125 
1 Abstract

Incorporating interactions into a biogeographical framework may serve to understand how interactions and the services they provide are distributed in space. We begin by simulating the spatiotemporal dynamics of realistic mutualistic networks inhabiting spatial networks of habitat patches. We proceed by comparing these predictions with the empirical results of a set of pollination networks in isolated hills of the Argentinian Pampas. We first find that one needs to sample up to five times as much area to record interactions as would be needed to sample the same proportion of species. Second, we find that peripheral patches have fewer interactions and harbor less nested networks — therefore potentially less resilient communities - compared to central patches. Our results highlight the important role played by the structure of dispersal routes on the spatial distribution of community patterns. This may help to understand the formation of biodiversity hotspots.

\section{KEYWORDS:}

Island biogeography, Metacommunities, Nestedness, Spatial networks, Species-area relationship, Pollination, Seed-dispersal 


\section{Introduction}

2 Biogeography attempts to describe the patterns of species distribution across

3 geographical areas and has a long tradition in ecology and evolutionary biology, with

4 early predecessors as influential as Alfred Russell Wallace. The publication of MacArthur

5 and Wilson's book on island biogeography (MacArthur \& Wilson, 1967) constituted a

6 formidable theoretical framework that brought a predictive ability to determine the

7 number of species on an island given its area and isolation. This, in turn, became a useful

\& tool in conservation biology, as for example in the problem of choosing between a single

9 large or several small (SLOSS) reserves (Higgs \& Usher, 1980; Simberloff \& Abele, 1976;

Diamond, 1975; Bascompte et al., 2007). The bridge to real-world conservation

11 applications has even become stronger around the concepts of metapopulations (Hanski,

12 1998) and metacommunites (Leibold et al., 2004). Despite this success, traditional island

13 biogeography dealt exclusively with species, ignoring their mutual dependencies. This

${ }_{14}$ approach resembles the development of the kinetic theory for ideal gases in physics, which

15 initially also neglected interactions (Alonso, Etienne \& McKane, 2006; Volkov et al.,

16 2007).

17 Almost independently of the above efforts in describing how species are distributed in

18 space, recent progress has made explicit that species interactions are an equally

19 important component of biodiversity. Interactions are at the core of significant ecosystem

20 services such as pollination or biological control. The way these interactions are arranged

${ }_{21}$ to create networks of interacting species largely affects the persistence of these networks

22 and the number of species they can support (Memmott, Waser \& Price, 2004; Burgos

23 et al., 2007; Okuyama \& Holland, 2008; Bastolla et al., 2009; Thébault \& Fontaine,

$\left.{ }_{24} 2010\right)$. In the context of global change, species interactions have also been found to be

25 even more susceptible than species themselves (Janzen, 1980; Tylianakis et al., 2008; 
1 Sabatino, Maceira \& Aizen, 2010). The disruption of interactions may in turn foresee

2 future species losses (Aizen, Sabatino \& Tylianakis, 2012).

3 A subsequent step, therefore, seems to be the inclusion of species interaction networks

4 into a biogeographical framework. An early contribution in this direction has been John

5 N. Thompson's geographic mosaic theory of coevolution (Thompson, 2005). This theory

6 has brought a geographic dimension to studies on the coevolution among free-living

7 species. For example, it has described how the sign and strength of interactions between

8 small groups of species depend on the local presence of other species. The influence of

9 both the geographic mosaic and early metapopulation theory lead to the development of metacommunity theory, which addresses the spatial distribution of small sets of

11 ecologically interacting species (Leibold et al., 2004). The first studies on

12 metacommunities were mainly theoretical descriptions of trophic modules, with very few

13 studies describing an entire, realistic ecological network. Only recently, there have been

14 significant steps in this direction (Olesen \& Jordano, 2002; Brose et al., 2004; Fortuna \&

15 Bascompte, 2006; Sabatino, Maceira \& Aizen, 2010; Sugiura, 2010; Gravel et al., 2011;

16 Pillai, Gonzalez \& Loreau, 2011; González-Castro, Traveset \& Nogales, 2012;

${ }_{17}$ Trø jelsgaard et al., 2013). As deviations from the behavior of ideal gases improved the

18 kinetic theory (Alonso, Etienne \& McKane, 2006; Volkov et al., 2007), consideration of

19 interactions has been shown to improve the accuracy of the predictions of the species-area

20 relationship (Brose et al., 2004; Gravel et al., 2011). So far, however, the focus has been

21 in correcting estimates of number of species (Brose et al., 2004; Gravel et al., 2011; Jabot

22 \& Bascompte, 2012), and unifying two previously unrelated scaling laws, i.e., the

23 species-area and the interaction-species relationships (Brose et al., 2004; Aizen, Sabatino

${ }_{24} \&$ Tylianakis, 2012). The few studies explicitly addressing mutualistic interactions among

25 species on a biogeographical scale have explored their dependence on island area

26 (Sugiura, 2010) or age (Trø jelsgaard et al., 2013). 
1 This exciting progress, therefore, still leaves unanswered the question of how interactions 2 and the networks they form are distributed across heterogeneous landscapes. A question

3 that, if answered, will provide insight on the relative role of regional and local processes in 4 shaping biodiversity. This would allow us, for instance, to achieve a better understanding 5 of where and how regional biodiversity hotspots are formed and maintained.

- Here we tackle this problem by using a metanetwork approach where species interaction 7 networks inhabit a spatial network of habitat patches (Fig. 1). We first present a general 8 framework based on extensive simulations. This allows controlling both the structure of 9 local interactions and that of spatial sites. We then compare these broad predictions with 10 the results for a particular case study formed by 12 pollination networks from untilled 11 hills or "sierras" raising in the Pampa region in Argentina (Sabatino, Maceira \& Aizen, 12 2010). This will show to what extent patterns predicted by our theoretical framework are 13 found in nature. In this context, we change the emphasis of biogeography from species to 14 their interactions, and look into the biogeographical patterns of such interactions.

${ }_{15}$ We ask whether interactions are relevant enough to modify conservation policies. For 16 that, we begin by answering the question of how many patches need to be conserved to 17 register a given fraction of species or interactions. Next, we explore how these 18 interactions are distributed across the different habitat patches. This has the potential to 19 be used as a prioritization tool. Finally, we scale all the way up to interaction networks to describe how network structure changes across the landscape. This later scale of 21 resolution will allow us to assess the interplay between spatial and local dynamics in 22 shaping regional patterns of biodiversity. 


\section{Materials and methods}

\section{Dispersal, colonization, and survival}

3 Metacomunity dynamics are simulated by a random process where species can colonize

4 empty patches and be driven locally extinct. Every time step is divided into a

5 colonization and an extinction phase. Roughly speaking, we use a spatially explicit

6 version of the model described in Fortuna \& Bascompte (2006). A patch is either empty

7 or occupied. The model has two parameters, a colonization and an extinction probability.

8 Patches where a certain species is absent can be colonized by that species with a

9 probability $c$. Similarly, a species present in a certain patch can go extinct with a

10 probability $e$.

11 The probability of a patch $p$ being colonized by species $s$ is given by the following

12 equation:

$$
C(p, s)=1-(1-c)^{a}
$$

13 where the exponent $a$ depends on whether species $s$ is an animal or a plant. If the former,

${ }_{14} a$ is equal to the number of adjacent patches where that animal is present. Adjacent

15 patches are those with a direct dispersal route to the focal patch $p$. Nevertheless, animals only live where they can find food. Therefore, if in patch $p$ there were none of the plant

17 species on which animal $s$ feeds, the probability of animal $s$ to colonize patch $p$ would be

18 zero. If species $s$ is a plant in a plant-pollinator network, the exponent $a$ is equal to the

19 number of adjacent patches where that plant is present.

Besides each animal species having a certain probability of becoming locally extinct, they go deterministically coextinct if every plant species they feed on has become extinct in that habitat patch.

${ }_{23}$ The extinction probability for animals is the same for all species and all patches 
$1 E(p, s)=e$. Regarding plants in plant-pollinator metacomunities, their extinction

2 probability depends on their number of pollinators present in that given patch $b$. This is

3 encapsulated in the following equation for a plant's extinction probability:

$$
E(p, s)=e / b
$$

4 Note that all probabilities are taken from a uniform distribution.

5 Despite the extreme simplicity behind these simulations, the model reproduces

6 qualitatively the patterns observed in nature. Thus, we can argue that these simple rules

7 are just enough to encapsulate the essence of the metacommunity dynamics.

\section{\& Spatial Networks}

9 We compare four different spatial structures of increasing heterogeneity in the number of links per habitat patch: a regular lattice, a random network (Erdös \& Rényi, 1959), an exponential network (following Barabasi \& Albert (1999) with uniformly random

preferential attachment). All theoretical results presented in main text correspond to the

random network, while we reproduce the equivalent results for the other spatial

topologies in the Appendix S1. This comparison serves to test to what degree spatial

structure affects the patterns here described. The advantage of using large theoretical

networks relies in the statistical power they provide and in our ability to control for

spatial heterogeneity.

The empirical dataset consists of 12 patches out of 18 from which we know the local plant-pollinator networks. The spatial network in this case is constructed by using a threshold distance under which two patches are considered to be linked trough dispersal (Urban \& Keitt, 2001). Here we use the thresholds that maximize the signal between the 
1 measured property and patch centrality (see Appendix S4 for details and an assessment

2 of the robustness of our analysis to this particular choice). In the construction of the

3 empirical spatial network we include all the 18 fragments. Although we do not sample

4 the other 6 , they can be part of one or many dispersal routes.

5 As a measure of the importance of a habitat patch within the spatial network, we focus

6 on its betweenness centrality. A patch's betweenness centrality measures the number of

7 times such a patch acts as a bridge along the shortest path between two other patches

8 (Freeman, 1977). This measure is intimately linked to dispersal within the network. Note

9 that this encapsulates the concept of isolation in MacArthur and Wilson's theory of

10 island biogeography. Thus, our work expands the few recent papers addressing networks

11 in a biogeographical dimension by adding a patch's isolation and spatial dynamics to the

12 previous focus on island area (Sugiura, 2010) and age (Trø jelsgaard et al., 2013).

\section{${ }_{13}$ Ecological Networks}

${ }_{14}$ The Pampean plant-pollinator networks were surveyed from 12 isolated "sierras," ranging

15 between 12 and 2147 ha and immersed in an intensively-used agricultural matrix. The number of plant species, animal species, and interactions ranged between 17 and 39, 48

17 and 79, and 132 and 243, respectively. Further details can be found in Sabatino, Maceira \& Aizen (2010) and Aizen, Sabatino \& Tylianakis (2012). For the simulations, we use the meta-web of these local networks, composed by 172 pollinators and 96 plants.

In our simulations, each patch of the spatial network harbors an empirical plant-pollinator network. It depicts the mutualistic interactions between plants and the animals that pollinate their flowers. The characteristics of those networks are described in Bascompte \& Jordano (2007). In particular, at the beginning of the simulation we populate each habitat fragment with the metaweb from Sabatino, Maceira \& Aizen (2010).

To see whether our results affect only plant-pollinator networks, or they are general to 
1 mutualistic interactions, we have also simulated the dynamics of plant-seed dispersers.

2 Both the details of the simulations and the results can be found in the Appendix S2. All

3 qualitative results shown here hold for this other set of simulations.

4 As a measure of overall network structure, here we focus on nestedness. In a nested

5 network, specialists interact with species that form well-defined subsets of the species

6 interacting with the most generalists (Bascompte et al., 2003). This is a pervasive

7 property of the architecture of mutualistic networks, with potential implications for their

\& persistence (Memmott, Waser \& Price, 2004; Burgos et al., 2007; Bastolla et al., 2009).

\section{Measure of Nestedness}

10 Nestedness is measured as in Bastolla et al. (2009), which has the advantage of being

11 related to network dynamics. Although there are other nestedness measures such as

${ }_{12}$ NODF (Almeida-Neto et al., 2008), they are all highly correlated and do not qualitatively

13 change our results (Bascompte \& Jordano, 2014). To measure relative nestedness, we

14 randomized the network a thousand times using the probabilistic model described in

15 Bascompte et al. (2003). Relative nestedness was then calculated as a $z$-score between the observed value and the randomizations (Eq. 3).

$$
z=\frac{N-\bar{N}_{r}}{\sigma_{N_{r}}},
$$

${ }_{17}$ where $\mathrm{N}$ is the nestedness value of the observed network, and $\bar{N}_{r}$ and $\sigma_{N_{r}}$ are the average

18 and standard deviation of nestedness values across randomized network replicates.

\section{${ }_{19}$ Results}

20 Our results show that one needs to sample a larger number of patches to record

21 interactions than would be necessary to record an equal proportion of species (Fig. 2). 
1 This difference is largely modulated by both environmental stochasticity - here

2 represented by the extinction-to-colonization ratio - and spatial heterogeneity - here

3 represented by the degree distribution of the spatial network (Appendix Fig. S1).

4 Specifically, the larger the environmental stochasticity, the larger the number of habitat

5 fragments that have to be sampled to achieve an equivalent proportion of interactions

6 (Appendix Fig. S2). This difference also grows with the heterogeneity of the spatial

7 network. Thus, for the random spatial network (Appendix Fig. S1) one would need to

8 sample 1.7 times more patches at the highest value of environmental stochasticity. This

9 difference grows up to 2.5 times more patches for the scale-free network (Appendix Figs. S2). In agreement with these theoretical results, the study of the empirical dataset shows

that - for this particular setting — one would need to sample twice the number of patches

12 to record $60 \%$ of the interactions than to record $60 \%$ of the species (Fig. 2).

13 Once we have shown how the total number of interactions scales up with number of

14 patches - that can be taken as surrogate of area in our model, we next move to mapping

15 how the number of interactions is distributed across the spatial network. This will inform

16 us on how the number of interactions depends on patch isolation. Our results show first

17 that more central patches - i.e., the more important ones in terms of dispersal across the

18 network (see Materials and Methods for a proper definition) - have more interactions and

19 more species than more isolated patches (as noted by the positive slopes in Fig. 3).

Second, the proportion of interactions in a patch-compared with the total number of interactions in the metaweb - is always lower than the equivalent proportion of species

${ }_{22}$ (as noted by the relative position of the two fitted lines in Fig. 3). This consistent pattern explains the previous result, i.e., the fact that the rarefaction curve of species lies above the rarefaction curve of interactions. This pattern is observed both in the

simulations and the analysis of the empirical dataset.

The above two results were based on counting the number of interactions and presenting 
1 this result either as an aggregated, or a patch-by-patch amount. We next consider the

2 patterning of these interactions in the context of how the nested structure of the local

3 mutualistic networks changes across space (Fig. 4). As shown by our results, central

4 patches sustain communities whose interactions are significantly nested, while peripheral

${ }_{5}$ patches harbor less nested communities with interactions organized in a way that does

6 not differ significantly from a random assembly. Indeed, the more central the habitat

7 patch is, the more significantly nested is its mutualistic community (Fig. 4). The same

8 pattern is found in the empirical metacommunity. In the Appendix S3 we show that the

9 relationship between nestedness and patch centrality is not mediated by the relationship

10 between network size and patch centrality. As also shown in the Appendix S5, patch

11 centrality is a better predictor of the number of interactions and of network structure

12 than patch area. However, the number of species in a patch is more correlated with patch

13 area than with patch centrality.

\section{${ }_{14}$ Discussion}

15 A predictive theory of how interactions are distributed in space may be relevant in the

16 context of understanding how habitat fragmentation will erode the architecture of

17 biodiversity and the services it provides. Specifically, knowing how this network structure

18 changes across the landscape can provide insight on how resilient these networks will be.

19 Similarly, it can guide efforts aimed at reversing this destructive trend, such as in reserve

20 design.

${ }_{21}$ The species-area relationship has been a cornerstone of a predictive conservation biology

22 in the sense of exploring how different re-arrangements of the habitat would translate into

23 different numbers of coexisting species. This can provide a rule of thumb in terms of how

${ }_{24}$ much area should be protected if we were interested in conserving a minimum number of 
1 species. In line with this, here we show that we may need as much as five times more

2 habitat patches in order to preserve a given proportion of total interactions, specially in

3 most fluctuating environments. This means that a reserve arrangement designed on the

4 basis of species presences and absences may not be enough to protect interactions, and

5 therefore will fail in protecting ecosystem functioning (Tylianakis et al., 2009).

6 In spatial networks of habitat patches, however, not all patches are equally relevant in

7 order to preserve interactions. We have shown that there is considerable variability in the

8 importance of a given patch in terms of harboring interactions and species. That

9 importance depends on the patch location in relation to the rest of the patches.

Therefore, this result should be interpreted in the light of the differential rescue effect across the landscape, which is linked to patch position. Even when the extinction rate of

12 a species was the same, the rescue effect by which a local patch can be recolonized from a

13 nearby patch is much lower in peripheral patches (Gilarranz \& Bascompte, 2012).

However, not only is the distribution of species heterogeneous across the landscape. As recently pointed out, in real landscapes there is not a random loss of interactions; while interactions between generalist species are ubiquitous, interactions involving specialist species are much more vulnerable (Aizen, Sabatino \& Tylianakis, 2012). Interestingly enough, this type of result can only be understood when integrating information on local network structure and landscape dynamics. This relates to two major theories bringing analytical tractability to multispecific coevolutionary studies: network theory (Bascompte \& Jordano, 2007) and the geographic mosaic theory of coevolution (Thompson, 2005). Beyond differences in the number of interactions across the landscape, different patches are also playing different roles in terms of how their interactions are assembled. Thus, the nested structure of the local network varies across the landscape. Given the importance of nestedness for maintaining network size (Bastolla et al., 2009) and robustness (Memmott, Waser \& Price, 2004; Burgos et al., 2007; Thébault \& Fontaine, 2010), local 
1 differences in nestedness are likely to translate into differences in the persistence of these

2 local networks. The higher stability of communities in central patches is twofold. First,

3 we have shown that central patches tend to harbor more nested communities. Other

4 things being equal, nested communities tend to be more persistent (Bastolla et al., 2009).

5 Second, this goes along the tendency for such central patches to be involved in a large

6 number of dispersal routes and therefore to have a higher incidence of rare species due to

7 a higher recolonization rate.

8 Network persistence, therefore, is a combination of local and regional processes that

9 cannot be understood independently from each other. The positive or negative feedbacks

10 between patch isolation and network structure may help to explain the spatial

11 distribution of biodiversity hotspots. These results pave the road for a deeper

12 understanding of how spatial structure and dispersal simultaneously affect local

13 community structure, the geographic distribution of ecosystem services, and

14 coevolutionary processes. 


\section{Acknowledgements}

We would like to thank M.A. Fortuna, D.B. Stouffer, R.P. Rohr, S. Saavedra, and V. Dakos for fruitful discussions. J. Tylianakis and L. Harder made useful suggestions to previous versions of this draft. Research was funded by the European Research Council through an Advanced Grant (to JB), the Spanish Ministry of Education trough a FPU PhD Fellowship (to LJG), and the National Institute of Agricultural Technology (INTA-PNECO1302), the Argentina National Research Council (CONICET-PIP 01623), the National Fund for Research (FONCyT-PICT 01300), and the National University of Comahue (UNC-B152/04) (to MS and MA).

\section{Data Accessibility}

Data will be made available in a public repository such as Dryad Digital Repository once the paper is accepted. However, it is accessible to reviewers upon request.

\section{References}

Aizen, M., Sabatino, M. \& Tylianakis, J. (2012) Specialization and Rarity Predict Nonrandom Loss of Interactions from Mutualist Networks. Science, 355, 1486-1489.

Almeida-Neto, M., Guimarães, P., Guimarães, J.P., Loyola, R. \& Ulrich, W. (2008) A consistent metric for nestedness analysis in ecological systems: reconciling concept and measurement. Oikos, 117, 1227-1239.

Alonso, D., Etienne, R. \& McKane, A. (2006) The merits of neutral theory. Trends in Ecology \& Evolution, 21, 451-457.

Barabasi, A.L. \& Albert, R. (1999) Emergence of scaling in random networks. Science, 286, 509-512.

Bascompte, J. \& Jordano, P. (2007) Plant-Animal Mutualistic Networks: The Architecture of Biodiversity. Annual Review of Ecology, Evolution, and Systematics, 38, 567-593. 
Bascompte, J. \& Jordano, P. (2014) Mutualistic Networks. Princeton University Press, New Jersey.

Bascompte, J., Jordano, P., Melián, C. \& Olesen, J. (2003) The nested assembly of plantanimal mutualistic networks. Proceedings of the National Academy of Sciences of the United States of America, 100, 9383-9387.

Bascompte, J., Luque, B., Olarrea, J. \& Lacasa, L. (2007) A probabilistic model of reserve design. Journal of Theoretical Biology, 247, 205-211.

Bastolla, U., Fortuna, M., Pascual-García, A., Ferrera, A., Luque, B. \& Bascompte, J. (2009) The architecture of mutualistic networks minimizes competition and increases biodiversity. Nature, 458, 1018-20.

Brose, U., Ostling, A., Harrison, K. \& Martinez, N. (2004) Unified spatial scaling of species and their trophic interactions. Nature, 428, 167-71.

Burgos, E., Ceva, H., Perazzo, R., Devoto, M., Medan, D., Zimmermann, M. \& Delbue, A. (2007) Why nestedness in mutualistic networks? Journal of Theoretical Biology, 249, $307-313$.

Diamond, J. (1975) The island dilemma: lessons of modern biogeographic studies for the design of natural reserves. Biological Conservation, 7, 129-146.

Erdös, P. \& Rényi, A. (1959) On random graphs I. Publicationes Mathematicae, 6, 290-297.

Fortuna, M. \& Bascompte, J. (2006) Habitat loss and the structure of plant-animal mutualistic networks. Ecology Letters, 9, 278-283.

Freeman, L. (1977) A set of measures of centrality based upon betweenness. Sociometry, $40,35-41$.

Gilarranz, L. \& Bascompte, J. (2012) Spatial network structure and metapopulation persistence. Journal of Theoretical Biology, 297, 11-16. 
González-Castro, A., Traveset, A. \& Nogales, M. (2012) Seed dispersal interactions in the Mediterranean Region: contrasting patterns between islands and mainland. Journal of Biogeography, 39, 1938-1947.

Gravel, D., Massol, F., Canard, E., Mouillot, D. \& Mouquet, N. (2011) Trophic theory of island biogeography. Ecology Letters, 14, 1010-6.

Hanski, I. (1998) Metapopulation dynamics. Nature, 396, 41-49.

Higgs, A. \& Usher, M. (1980) Should nature reserves be large or small? Nature, 285, $568-569$.

Jabot, F. \& Bascompte, J. (2012) Bitrophic interactions shape biodiversity in space. Proceedings of the National Academy of Sciences of the United States of America, 109, $4521-6$.

Janzen, D. (1980) When is it coevolution? Evolution, 34, 611-12.

Leibold, M., Holyoak, M., Mouquet, N., Amarasekare, P., Chase, J.M., Hoopes, M.F., Holt, R.D., Shurin, J.B., Law, R., Tilman, D., Loreau, M. \& Gonzalez, A. (2004) The metacommunity concept: a framework for multi-scale community ecology. Ecology Letters, 7, $601-613$.

MacArthur, R. \& Wilson, E. (1967) The Theory of Island Biogeography. Princeton University Press, New Jersey.

Memmott, J., Waser, N. \& Price, M. (2004) Tolerance of pollination networks to species extinctions. Proceedings of the Royal Society B, 271, 2605-2611.

Okuyama, T. \& Holland, J. (2008) Network structural properties mediate the stability of mutualistic communities. Ecology Letters, 11, 208-216.

Olesen, J. \& Jordano, P. (2002) Geographic patterns in plant-pollinator mutualistic networks. Ecology, 83, 2416-2424. 
Pillai, P., Gonzalez, A. \& Loreau, M. (2011) Metacommunity theory explains the emergence of food web complexity. Proceedings of the National Academy of Sciences of the United States of America, 108, 19293-8.

Sabatino, M., Maceira, N. \& Aizen, M. (2010) Direct effects of habitat area on interaction diversity in pollination webs. Ecological Applications, 20, 1491-1497.

Simberloff, D. \& Abele, L. (1976) Island biogeography theory and conservation practice. Science, 191, 285-286.

Sugiura, S. (2010) Species interactions-area relatioships: biological invasions and network structure in relation to island area. Proceedings of the Royal Society B, 277, 1807-1815.

Thébault, E. \& Fontaine, C. (2010) Stability of ecological communities and the architecture of mutualistic and trophic networks. Science, 329, 853-6.

Thompson, J. (2005) The Geographic Mosaic of Coevolution. The University of Chicago Press.

Trø jelsgaard, K., Báez, M., Espadaler, X., Nogales, M., Oromí, P., Roche, F.L. \& Olesen, J.M. (2013) Island biogeography of mutualistic interaction networks. Journal of Biogeography, 40, 2020-2031.

Tylianakis, J., Laliberté, E., Nielsen, A. \& Bascompte, J. (2009) Conservation of species interaction networks. Biological Conservation, 143, 2270-2279.

Tylianakis, J., Didham, R., Bascompte, J. \& Wardle, D. (2008) Global change and species interactions in terrestrial ecosystems. Ecology Letters, 11, 1351-1363.

Urban, D. \& Keitt, T. (2001) Landscape connectivity: a graph-theoretic perspective. Ecology, 85, 1205-1218.

Volkov, I., Banavar, J., Hubbell, S. \& Maritan, A. (2007) Patterns of relative species abundance in rainforests and coral reefs. Nature, 250, 45-49. 


\section{The following Supporting Information is available for this article online}

Appendix S1: Plant-pollinator interactions.

Appendix S2: Seed-disperser interactions.

Appendix S3: Nestedness increases with patch centrality independently of network size and connectance.

Appendix S4: Criteria to create the empirical spatial network.

Appendix S5: Influence of patch centrality and area. 


\section{Figure captions}

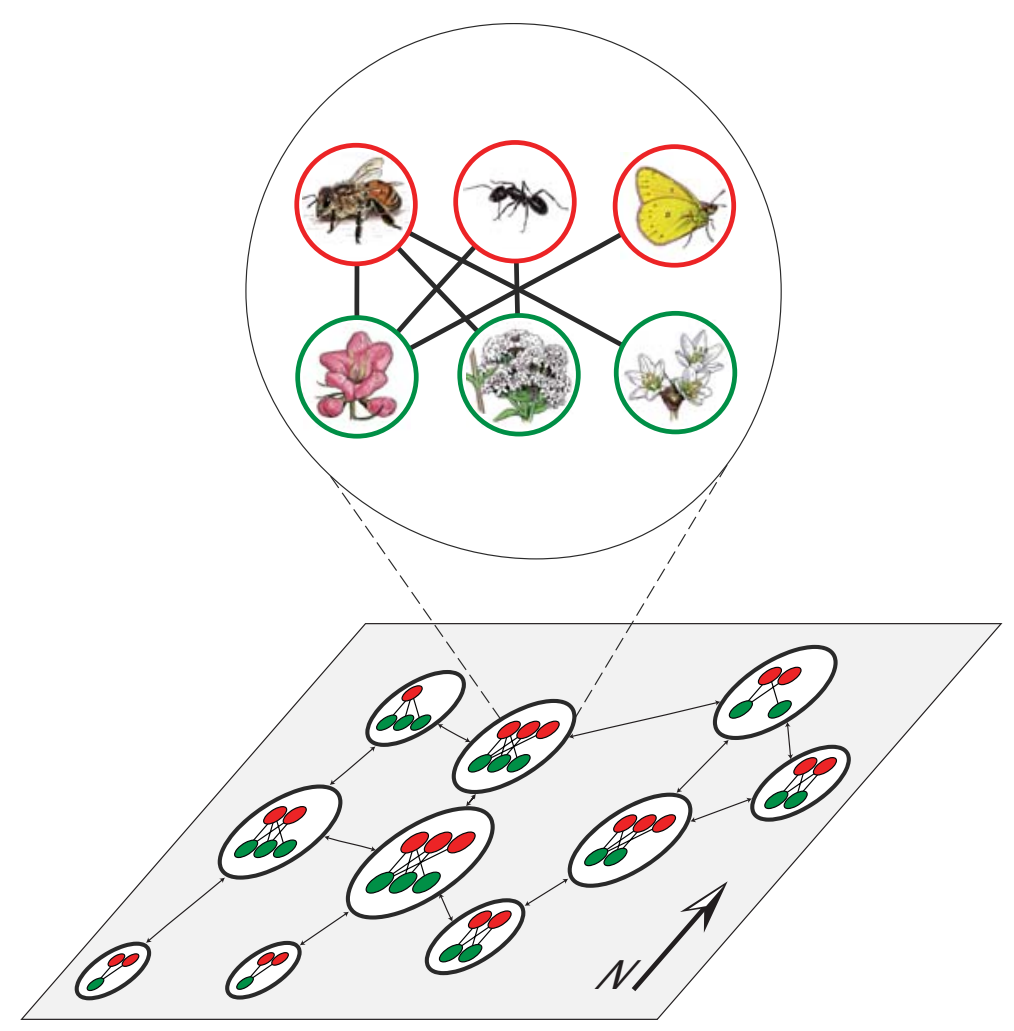

Figure 1: Schematic representation of the theoretical framework developed in this manuscript. Each patch of the spatial network (bottom) harbors a local mutualistic network (top). The figure depicts a subset of the metaweb described in Sabatino, Maceira \& Aizen (2010). Pollinator species are, from left to right, Apis mellifera, Camponotus sp, and Colias lesbia. Plant species are, from left to right, Gerardia genistifolia, Stevia satureiifolia, and Nothoscordum bonariense. Drawings by J. López Rojas. 

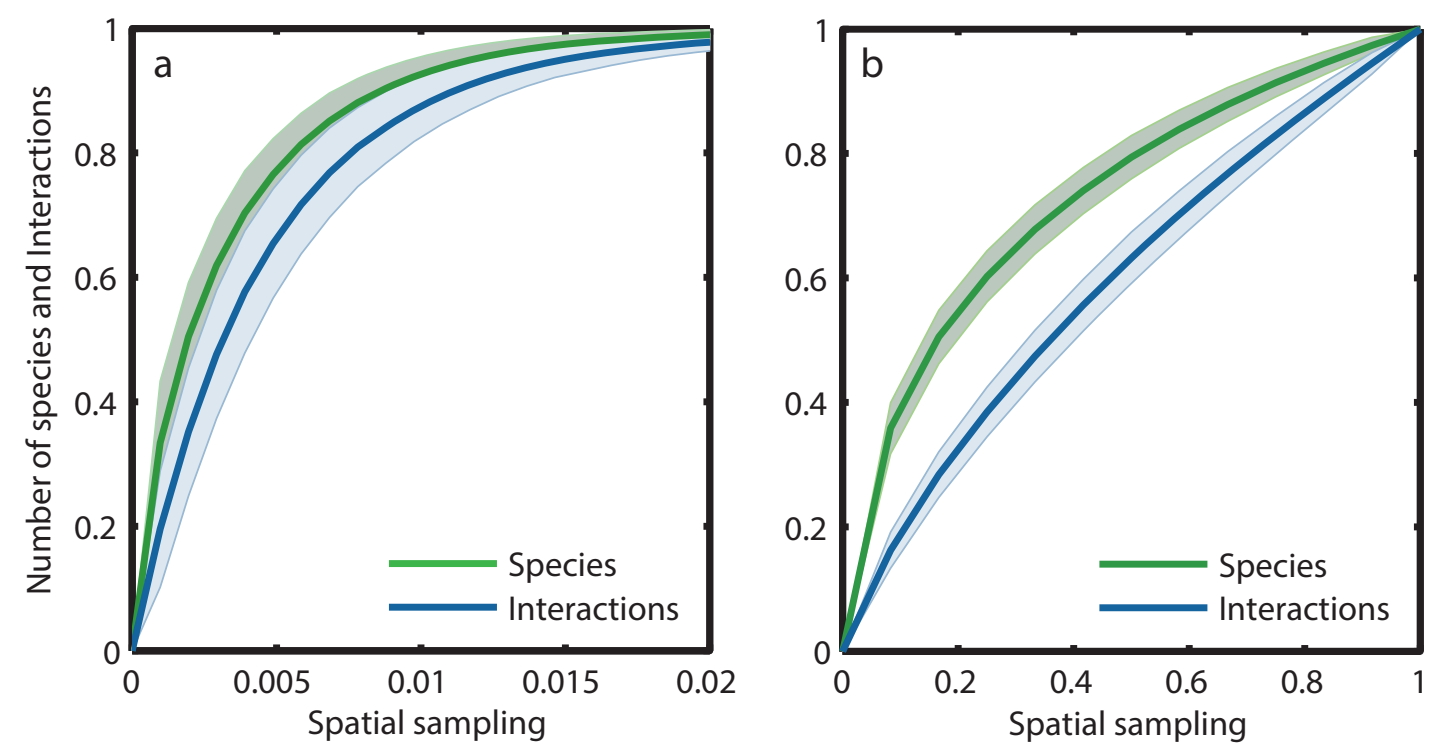

Figure 2: Rarefaction curves of (a) species (green) and (b) interactions (blue) for the metacommunity simulations, and for the empirical case study. Spatial sampling is measured as the proportion of sampled patches. Here we use the proportion of sampled species and interactions, respectively, in order to obtain comparable magnitudes. To obtain panel (a) the model runs in a spatial network with a random degree distribution, 1,024 nodes, and an extinction-to-colonization ratio of 2.2. After 10,000 time steps, we randomly sample the patches and count the new species and interactions found. Panel (b) corresponds to the empirical case study. The empirical network has 18 nodes. The habitat patches are also selected randomly. Shadowed areas represent the $95 \%$ confidence interval. 

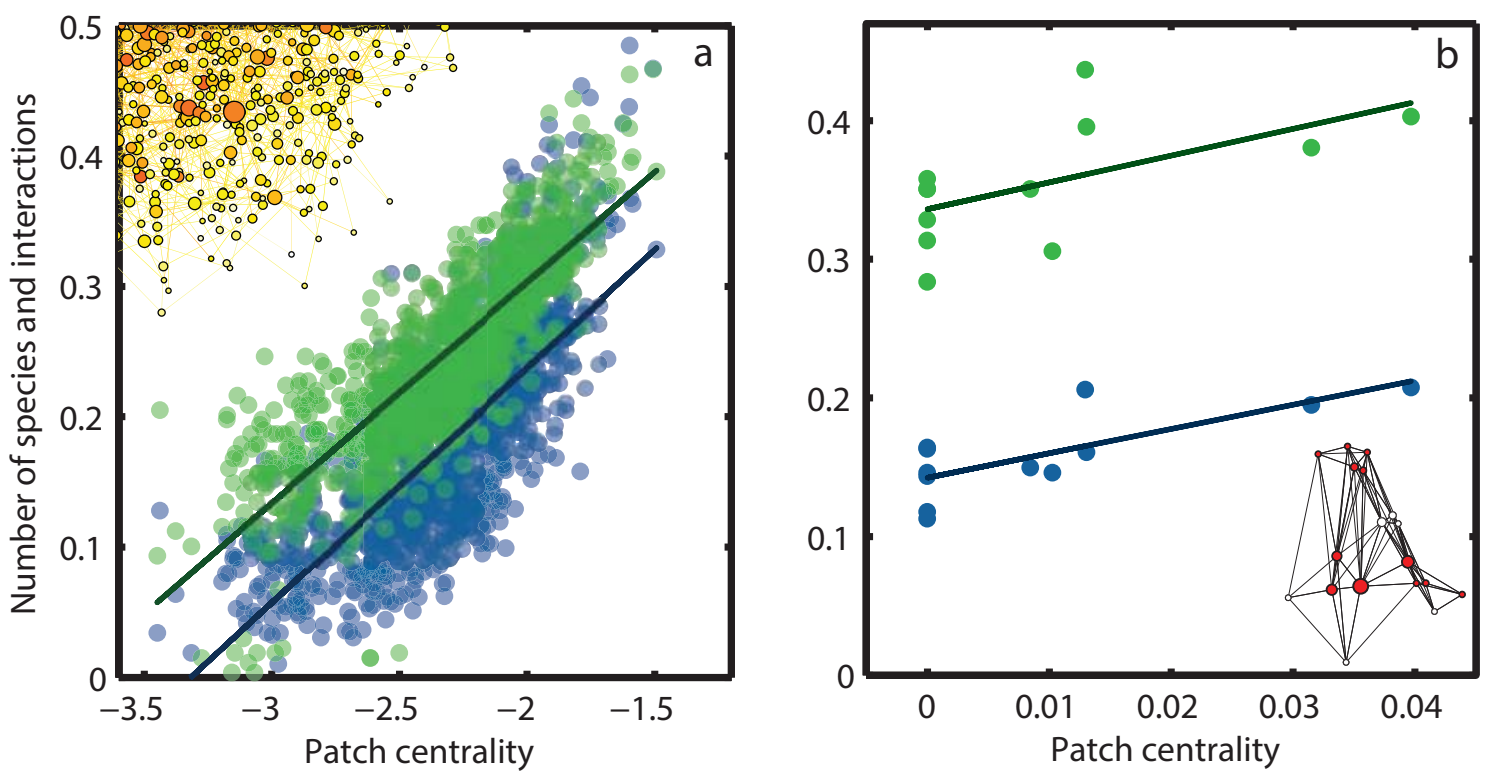

Figure 3: Spatial distribution of species and interactions for the simulated (a) and empirical (b) metacommunities. (a) The normalized number of species (green) and interactions (blue) is significantly correlated with the log betweenness centrality of the patch where the community is harbored (Spearman correlation, $p<0.001, r=0.80$ for interactions; and $p<0.001, r=0.85$ for species). In the top-left corner is shown a fragment of the theoretical network of 1,024 patches with a random degree distribution, and an extinction-to-colonization ratio of 2.2 . The size of the nodes is proportional to their betweenness centrality, and the color is redder the larger is the number of interactions found in that patch. (b) The same result for the empirical case study $(p=0.016, r=0.67$ for interactions; and $p=0.019, r=0.66$ for species). The spatial network of the 18 patches with a threshold distance of $17 \mathrm{Km}$ is represented in the bottom-right corner. Patches that were actually sampled are represented in red. The normalized number of species and interactions is calculated comparing the number found in the focal patch with the number of species or interactions found in the metaweb (Sabatino, Maceira \& Aizen, 2010). Fitted lines are only meant to guide the eye. 

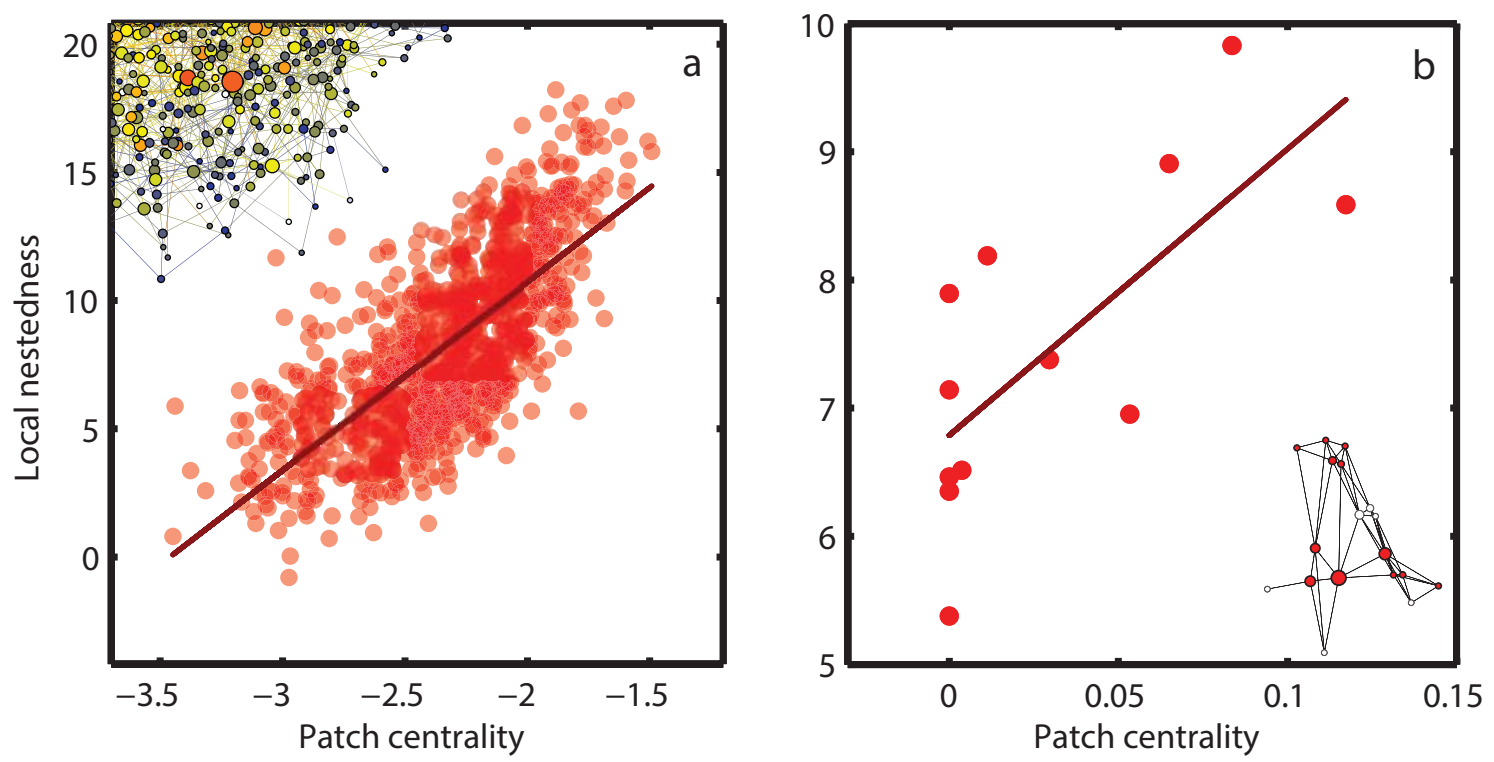

Figure 4: Spatial distribution of network structure for the simulated (a) and empirical (b) metacommunities. The average relative nestedness of a local community is significantly correlated with the log betweenness centrality of the patch where the community is harbored (a) (Spearman correlation, $p<0.001, r=0.74$ ). The results in here represent a random spatial network of 1,024 patches with an extinction-to-colonization ratio of 2.2. In the top-left corner we show a fragment of that spatial network. The size of the nodes is proportional to their betweenness centrality. White color indicates non-significantly nested networks (z-score $\in[-1.96, \cdots, 1.96])$. Blue/yellow/red scale represents significantly nested communities. The redder the node, the larger the nestedness of the community it harbors. (b) The same result for the empirical case study (Spearman correlation, $p=0.005, r=0.75)$. The spatial network of the 18 patches with a threshold distance of $12 \mathrm{Km}$ is represented in the bottom-right corner. Patches that were actually sampled are represented in red. Fitted lines are only meant to guide the eye. 